\title{
Preoperative Localization in Colonic Surgery (PLoCoS Study): a multicentric experience on behalf of the Italian Society of Colorectal Surgery (SICCR)
}

\author{
Michele Manigrasso $^{1}$ (D) Marco Milone ${ }^{2} \cdot$ Mario Musella $^{1} \cdot$ Pietro Venetucci $^{1} \cdot$ Francesco Maione $^{2} \cdot$ Ugo Elmore $^{3}$. \\ Gaetano Gallo ${ }^{4}$. Roberto Perinotti ${ }^{5}$. Giovanni Domenico De Palma ${ }^{2} \cdot$ PLoCoS Study Group
}

Received: 4 August 2021 / Accepted: 19 September 2021 / Published online: 5 October 2021

(c) The Author(s) 2021

\begin{abstract}
The aim of this prospective multicentric study was to compare the accurate colonic lesion localization ratio between CT and colonoscopy in comparison with surgery. All consecutive patients from 1st January to 31 st December 2019 with a histologically confirmed diagnosis of dysplastic adenoma or adenocarcinoma with planned elective, curative colonic resection who underwent both colonoscopy and CT scans were included. Each patient underwent conventional colonoscopy and CT to stage the tumour, and the localization results of each procedure were registered. CT and colonoscopic localization were compared with surgical localization, adopted as the reference. Our analysis included 745 patients from 23 centres. After comparing the accuracy of colonoscopy and CT (for visible lesions) in localizing colonic lesions, no significant differences were found between the two preoperative tools ( $510 / 661$ vs 499/661 correctly localized lesions, $p=0.518$ ). Furthermore, after analysing only the patients who underwent complete colonoscopy and had a visible lesion on CT, no significant difference was observed between conventional colonoscopy and CT (331/427 vs 340/427, $p=0.505)$. Considering the intraoperative localization results as a reference, a comparison between colonoscopy and CT showed that colonoscopy significantly failed to correctly locate the lesions localized in the descending colon (17/32 vs $26 / 32, p=0.031)$. We did not identify an advantage in using CT to localize colonic tumours. In this setting, colonoscopy should be considered the reference to properly localize lesions; however, to better identify lesions in the descending colon, CT could be considered a valuable tool to improve the accuracy of lesion localization.
\end{abstract}

Keywords Colorectal $\cdot$ Colonoscopy $\cdot$ CT scan $\cdot$ Localization $\cdot$ Colon cancer $\cdot$ Surgery

Members of the "PLoCoS Study group" are listed in acknowledgement section.

Michele Manigrasso

michele.manigrasso@unina.it

1 Department of Advanced Biomedical Sciences, "Federico II" University of Naples, Via Sergio Pansini 5, 80131 Naples, Italy

2 Department of Clinical Medicine and Surgery, "Federico II" University of Naples, Via Pansini 5, 80131 Naples, Italy

3 Division of Gastrointestinal Surgery, San Raffaele Scientific Institute, 60 Via Olgettina, 20132 Milan, Italy

4 Operative Unit of General Surgery, Department of Medical and Surgical Sciences, University of Catanzaro, Catanzaro, Italy

5 Colorectal Surgical Unit, Department of Surgery, Infermi Hospital, Biella, Italy

\section{Introduction}

Although colonoscopy is currently considered the method of choice to detect colorectal cancer, little is known about its accuracy in tumour localization $[1,2]$.

In fact, colonoscope orientation throughout the colonic segments is complicated by the absence of specific anatomic landmarks between the anal verge and the ileocecal valve, resulting in inaccurate lesion localization in $11-21 \%$ of cases [3-6].

Inaccurate localization plays a critical role during planned surgical procedures, especially laparoscopic and robotic surgery, in which trocar positioning or arms docking are fundamental to performing the correct surgical intervention [7-9].

In this setting, computed tomography (CT) could be considered another approach for correctly localizing colonic 
lesions, as it is able to locate the major colonic anatomical landmarks, caecum and colonic flexures [10].

The aim of this prospective multicentric study was to compare $\mathrm{CT}$ and colonoscopy in terms of their accuracy in localizing colonic lesions.

\section{Materials and methods}

The study was approved by our institutional review board, and informed consent was obtained from all subjects before enrolment. A 1-year prospective observational study enrolling all consecutive patients in tertiary referral colorectal centres from 1st January to 31st December 2019 with a histologically confirmed diagnosis of colon dysplastic lesion or cancer was performed. The study findings have been reported in compliance with the STROBE checklist [11].

All patients with a histologically confirmed diagnosis of dysplastic adenoma or adenocarcinoma with planned elective, curative colonic resection who underwent both colonoscopy and CT were included. The exclusion criteria were as follows: rectal cancer detected during preoperative staging; inability to perform preoperative colonoscopy or CT; emergency surgery; curative endoscopic treatment; and death before surgery.

Each patient underwent conventional colonoscopy and CT to stage the tumour, and the localization results of each procedure were registered. CT and colonoscopic localization were compared with surgical localization, which was adopted as the reference standard.

Colonoscopic localization was performed by an expert endoscopist (at least 500 colonoscopies per year). Furthermore, an endoscopist indicated the colon tract affected by the lesion, choosing among eight segments as shown below.

CT exams were performed by an expert radiologist (at least $300 \mathrm{CT}$ scans per year) with multislice devices and at least 64 slices.

Briefly, first, a "scout exam" of the abdomen and the pelvis was performed, followed by a multiphasic CT study. The latter was first performed without contrast medium and then during the venous phase after an injection of iodate contrast medium. In both phases, with and without contrast, the whole abdomen and pelvis were checked to be sure that the colon was completely included. In selected cases, integrative scans were performed, especially if patients already underwent colonic surgery. The radiologist indicated the colon tract in which the lesion was present, choosing among the colonic segments as shown below.

The endoscopists and radiologists were blinded to the lesion localization results.

Finally, the lesion location was intraoperatively identified by the surgeon. The type of intervention (open surgery or minimally invasive approach) was chosen by each surgeon according to his/her preferences.

In each procedure, the colon was divided into 8 parts (Fig. 1):

- Rectosigmoid junction: between the last Houston valve and the first tract of the sigmoid segment;

- Sigmoid: tortuous segment between 40 and $15 \mathrm{~cm}$ from the anus (last Houston valve);

- Descending colon: straight segment between $10 \mathrm{~cm}$ from the splenic flexure and $40 \mathrm{~cm}$ from the anus;

- Splenic flexure: tract between $10 \mathrm{~cm}$ before and after the splenic curve of the colon;

- Transverse colon: tract between $10 \mathrm{~cm}$ after the splenic flexure and $10 \mathrm{~cm}$ before the hepatic flexure;

- Hepatic flexure: tract between $10 \mathrm{~cm}$ before and after the hepatic curve of the colon;

- Ascending colon: tract between $10 \mathrm{~cm}$ proximal from the hepatic curve and ileocecal valve;

- Caecum: tract limited by the ileocecal valve and the bottom of the caecum with the appendix orifice.

\section{Data analysis and outcomes assessment}

For the included patients, data on sex, age, body mass index (BMI), American Society of Anaesthesiologists (ASA) score and previous colonic surgery were recorded.

During colonoscopy, in addition to lesion localization, data about the colonoscopy procedure and the presence of an obstructing mass were recorded. During the CT scans, data about the visibility of the lesion were recorded. Intraoperative data included the type of intervention (open, laparoscopic, or robotic approach), the need to modify the surgical approach because of an incorrect lesion localization and the type of the modified approach.

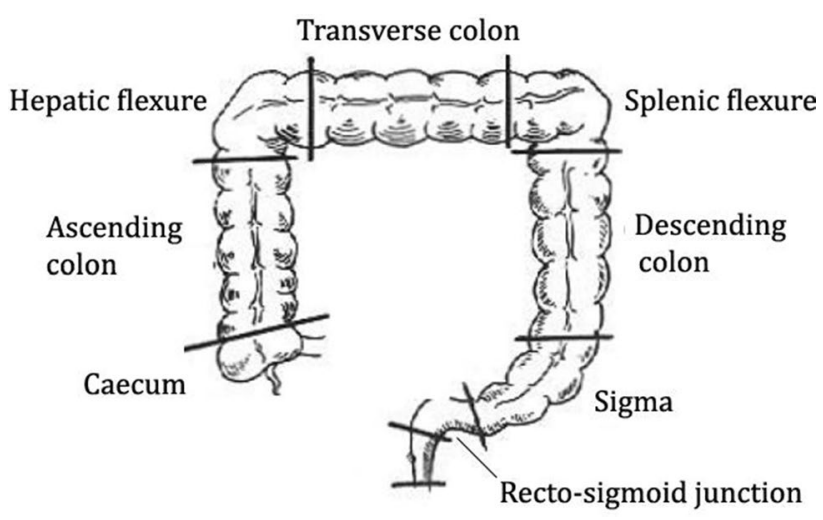

Fig. 1 Division of the colon in eight segments 
Finally, the tumour characteristics included $\mathrm{T}$ stage and the maximum diameter of the lesion, expressed in centimetres $(\mathrm{cm})$.

The primary outcome was the accurate lesion localization ratio of conventional colonoscopy and computed tomography in localizing colonic lesions, in comparison with surgery.

Accurate localization was defined as the ratio of colonoscopic or imaging localization with intraoperative localization (considered the true value) and was expressed in percentage.

The secondary outcome was patient and disease characteristics (age, sex, BMI, previous colonic surgery, T stage and tumour size) that influenced correct localization of the lesion.

\section{Statistical analysis}

Statistical analyses were performed with SPSS 26.0 (SPSS Inc., IBM, Chicago, IL, USA).

Continuous variables are expressed as the mean \pm standard deviation (SD); categorical variables are expressed as percentages (\%). Continuous variables were compared by the Mann-Whitney $U$ test and $t$-test, and categorical variables were compared by the Chi-square test. When the minimum expected value was less than five, we adopted Fisher's exact test. A $p$ value $<0.05$ was defined as statistically significant.

The agreement between the two diagnostic methods was calculated using the weighted Cohen $\kappa$ statistics. The $\kappa$ values were considered as follows: 0-0.20, slight agreement; 0.21-0.40, fair agreement; 0.41-0.60, moderate agreement; $0.61-0.80$, substantial agreement; and $0.81-1$ almost perfect agreement.

A multivariate analysis (stepwise method) was adopted to identify tumour and patient factors independently associated with incorrect lesion localization by each preoperative procedure, expressed by the odds ratio (OR) and $95 \%$ confidence interval $(95 \% \mathrm{CI})$.

\section{Results}

Our analysis included 745 patients from 23 centres (22 across Italy and one in France).

Of these patients, one was excluded because he died before CT, seven patients were excluded because of the rectal location of the neoplasia during preoperative staging, two were excluded because of the inability to perform colonoscopy (no compliance with bowel preparation), and six patients were excluded because the lesion was endoscopically treated. Thus, the final analysis involved 729 patients.

\section{Demographic and pathological data}

Of the 729 included patients, $411(56.4 \%)$ were male, the mean age was $70 \pm 11.21$ years, the mean ASA score was $2.36 \pm 0.64$, and the mean BMI was $25.84 \pm 4.3 \mathrm{~kg} / \mathrm{m}^{2} ; 22$ patients $(3 \%)$ underwent previous colonic resection. Regarding the pathological data, the lesions were dysplastic adenoma or adenocarcinoma in situ in $4.4 \%$ of cases, stage $\mathrm{T} 1$ in $8.8 \%$, stage $\mathrm{T} 2$ in $15.8 \%$, stage $\mathrm{T} 3$ in $50.8 \%$, stage $\mathrm{T} 4$ in $15.9 \%$, and not reported in $4.4 \%$.

The maximum diameter of the lesion (dmax) was reported in 677 cases, with a mean dmax of $4.4 \pm 2.16 \mathrm{~cm}$. The demographic and pathological data are shown in Table 1.

\section{Lesion localization by colonoscopy}

Colonoscopy was completed in 488 cases (66.9\%). The reasons for incomplete colonoscopy were tumoral stenosis $(88.8 \%)$, inadequate colon cleansing $(2.5 \%)$, lack of patient compliance $(0.8 \%)$, intraoperative bleeding $(0.4 \%)$, and unreported reasons $(7.5 \%)$.

Colonoscopy localized the lesions throughout the eight colonic segments, with most lesions located in the sigmoid (26.6\%), ascending colon $(21.1 \%)$ and caecum $(15.1 \%)$. Comparing intraoperative and colonoscopic localization,

Table 1 Demographic characteristics of the included patients and pathologic data of the lesions

\begin{tabular}{ll}
\hline Characteristics & No of patients $(\%)$ \\
\hline Patients & 729 \\
Male & $441(56.4)$ \\
Female & $318(43.6)$ \\
Age (years) & $70 \pm 11.21$ \\
BMI $\left(\mathrm{kg} / \mathrm{m}^{2}\right)$ & $25.84 \pm 4.3$ \\
ASA score & $2.36 \pm 0.64$ \\
I & $50(6.9)$ \\
II & $364(49.9)$ \\
III & $277(38)$ \\
IV & $15(2.1)$ \\
Not reported & $23(3.2)$ \\
Previous colonic resection & $22(3)$ \\
T stage & \\
Dysplastic adenoma/ T in situ & $32(4.4)$ \\
T1 & $64(8.8)$ \\
T2 & $115(15.8)$ \\
T3 & $370(50.8)$ \\
T4 & $116(15.9)$ \\
Not reported & $32(4.4)$ \\
Lesion dmax & $4.4 \pm 2.16 \mathrm{~cm}$ \\
\hline
\end{tabular}

$B M I$ body mass index, dmax maximum diameter, $\mathrm{cm}$ centimetres 
there were small differences in all the colonic segments, but the differences were not significant.

Correct localizations were obtained in 544 cases $(74.6 \%)$. The results of lesion localization by colonoscopy are summarized in Table 2.

\section{Lesion localization by CT}

Preoperative CT visualized a colonic lesion in $90.7 \%$ of cases (661/729). Correct localization was reported in $70.1 \%$ of cases (519/729). However, when only the 661 cases in which the lesion was visualized was considered, the accuracy increased to $77.2 \%(510 / 661)$.

Of the 185 lesions erroneously localized by colonoscopy, 74 lesions (40\%) were accurately localized by CT.

Thus, combining the correct CT localization with the incorrect colonoscopic localization, the combined accuracy reached $84.8 \%$, localizing 618 of 729 lesions.

Similarly, on CT scans, most lesions were localized in the sigmoid (25.9\%), ascending colon (20.9\%) and caecum (17.7\%).

In the comparison of imaging and intraoperative data, small nonsignificant differences were recorded in each colonic segment. The data on lesion localization by $\mathrm{CT}$ are reported in Table 3.

Table 2 Colonoscopy data, lesion localization and comparison with intraoperative localization

\begin{tabular}{lccc}
\hline Characteristics & $\begin{array}{c}\text { Colonoscopy } \\
n=729(\%)\end{array}$ & Surgery $n=729(\%)$ & $p$ value \\
\hline Localization & & & \\
Caecum & $110(15.1)$ & $124(17)$ & 0.354 \\
Ascending colon & $154(21.1)$ & $157(21.5)$ & 0.896 \\
Hepatic flexure & $67(9.2)$ & $49(6.7)$ & 0.858 \\
Transverse colon & $62(8.5)$ & $70(9.6)$ & 0.523 \\
Splenic flexure & $43(5.9)$ & $54(7.4)$ & 0.293 \\
Descending colon & $57(7.8)$ & $41(5.6)$ & 0.176 \\
Sigma & $194(26.6)$ & $184(25.2)$ & 0.591 \\
Recto-sigmoid junc- & $42(5.8)$ & $49(6.7)$ & 0.516 \\
$\quad$ tion & & & \\
Overall accuracy & $544(74.6)$ & & \\
Incomplete colonos- & $241(33.1)$ & & \\
$\quad$ copy & & & \\
Reason for incomplete colonoscopy & & \\
Tumoral stenosis & $214(88.8)$ & & \\
Inadequate colon & $6(2.5)$ & & \\
$\quad$ cleansing & & \\
No patient compli- & $2(0.8)$ & & \\
ance & & & \\
Intraluminal bleeding & $1(0.4)$ & & \\
Not reported & $16(7.5)$ & & \\
\hline
\end{tabular}

Table 3 CT scan data, lesion localization and comparison with intraoperative localization

\begin{tabular}{llcc}
\hline Characteristics & CT scan $(\%)$ & Surgery (\%) & $p$ value \\
\hline Number of detected lesions & $661(90.7)$ & & \\
Localization & & & \\
Caecum & $117(17.7)$ & $118(17.8)$ & $1.000^{*}$ \\
Ascending colon & $138(20.9)$ & $144(21.8)$ & $0.687^{*}$ \\
Hepatic flexure & $59(8.9)$ & $42(6.3)$ & $0.151^{*}$ \\
Transverse colon & $54(8.1)$ & $65(9.9)$ & $0.337^{*}$ \\
Splenic flexure & $42(6.4)$ & $52(7.9)$ & $0.335^{*}$ \\
Descending colon & $58(8.8)$ & $32(4.8)$ & $0.083^{*}$ \\
Sigma & $171(25.9)$ & $161(24.4)$ & $0.568^{*}$ \\
$\quad$ Recto-sigmoid junction & $32(4.8)$ & $47(7.1)$ & $0.104^{*}$ \\
Overall accuracy & $510(70.1)$ & & \\
Accuracy on detected lesions & $510 / 661(77.2)$ & & \\
\hline
\end{tabular}

*Analyses are performed on 661 patients (lesions seen at CT)

\section{Comparison of colonoscopy and computed tomography}

When comparing the accurate lesion localization ratio of colonoscopy and CT (for visible lesions), no significant differences were found between the two preoperative tools (510/661 vs 499/661 correctly localized lesions, $p=0.518$ ).

Furthermore, analysing only the patients who underwent complete colonoscopy with a lesion visible on CT, the comparison of colonoscopy and computed tomography showed no significant difference in correct lesions localization (331/427 vs $340 / 427$ correctly localized lesions, $p=0.505$ ).

Considering the intraoperative localization as a reference, a comparison between colonoscopy and CT showed that colonoscopy significantly failed to correctly locate the lesions in the descending colon (17/32 vs $26 / 32, p=0.031)$.

However, the agreement between the two methods in comparison with the intraoperative findings demonstrated an almost perfect agreement between the two procedures (CT scan versus colonoscopy, weighted $\kappa: 0.881$ ).

The comparison between colonoscopy and computed tomography in accurate lesions localization is reported in Table 4.

\section{Intraoperative data}

Open surgery was performed in 180 patients (24.8\%), while laparoscopy was performed in $529(72.6 \%)$ and roboticassisted surgery was performed in 19 (2.6\%).

Among the cases in which colonoscopy or both methods incorrectly localized the lesions, a change in intraoperative management was necessary in 29 cases (4\%). In fact, 4 minimally invasive procedures were converted to open surgery; 
Table 4 Comparison between colonoscopy lesion localization and CT scan

\begin{tabular}{lccc}
\hline Localization (intraoperative) & Colonoscopy & CT scan & $p$ value \\
\hline Accurate localization & 499 & 510 & $0.518^{*}$ \\
Localization & & & \\
Caecum (118) & 100 & 106 & 0.328 \\
Ascending colon (144) & 114 & 116 & 0.883 \\
Hepatic flexure (42) & 28 & 31 & 0.633 \\
Transverse colon (65) & 36 & 38 & 0.859 \\
Splenic flexure (52) & 29 & 29 & 1.000 \\
Descending colon (32) & 17 & 26 & $\mathbf{0 . 0 3 1}$ \\
Sigma (161) & 141 & 137 & 0.627 \\
Recto-sigmoid junction (47) & 34 & 27 & 0.194 \\
\hline
\end{tabular}

*Analyses are performed on 661 patients (lesions seen at CT)

in three cases, intraoperative colonoscopy was needed, and in the other 22 cases, the planned resection was modified.

Considering the real localization of the lesions, in the 4 converted cases the error differed by two colonic segments (ascending colon vs middle transverse colon); in the other remaining 25 cases the localization error differed by only one segment (right colon vs transverse and left colon vs recto-sigmoid junction/splenic flexure).

The intraoperative data are shown in Table 5.

\section{Multivariate analyses}

Multivariate analyses showed that neither colonoscopy and CT were significantly influenced by any of the patients' characteristics or pathological data. The results of the multivariate analysis are shown in Table 6.

\section{Discussion}

To the best of our knowledge, this is the largest series to compare CT and conventional colonoscopy in lesion localization.

Correct preoperative lesion localization is one of the most important aspects for optimal preoperative surgical planning.

In fact, incorrect localization is a cause of on-table alterations in surgical management, especially in minimally invasive surgery, leading to the need for an additional trocar or a different type of robotic docking $[9,12]$.

Table 5 Changes in on-table management

\begin{tabular}{llll}
\hline Planned & Modified & Reason & No of cases \\
\hline Right hemicolectomy & Extended right hemicolectomy & Lesion of the transverse & 5 \\
& Conversion to open & lesion of the transverse, technical difficulties & 4 \\
& Transverse colon resection & Lesion in middle transverse & 1 \\
Left hemicolectomy & Splenic flexure resection & Lesion in the splenic flexure & 5 \\
& Extended left hemicolectomy & Lesion in the transverse & 2 \\
& Intraoperative coloscopy & Lesion in the splenic flexure/transverse & 5 \\
& Anterior resection with AMI preserving & Lesion of the recto-sigmoid junction & 1 \\
\hline
\end{tabular}

IMA inferior mesenteric artery

Table 6 Multivariate analyses

\begin{tabular}{lll}
\hline Factors & Colonoscopy $p$ value $(\mathrm{OR} ; 95 \%$ CI $)$ & CT scan $p$ value $(\mathrm{OR} ; 95 \%$ CI $)$ \\
\hline Age & $0.59(0.981 ; 0.962,1.001)$ & $0.129(0.985 ; 0.967,1.004)$ \\
BMI & $0.684(1.010 ; 0.964,1.058)$ & $0.822(0.995 ; 0.950,1.041)$ \\
Gender & $0.956(1.011 ; 0.679,1.505)$ & $0.442(1.165 ; 0.789,1.719)$ \\
ASA score & $0.931(1.016 ; 0.716,1.440)$ & $0.080(1.345 ; 0.965,1.875)$ \\
Previous colonic resection & $0.428(1.557 ; 0.521,4.654)$ & $0.702(0.779 ; 0.216,2.803)$ \\
Obstructing mass & $0.359(0.653 ; 0.263 ; 1.622)$ & $\mathrm{NP}$ \\
Bowel preparation & $0.839(0.978 ; 0.786,1.216)$ & $\mathrm{NP}$ \\
Complete colonoscopy & $0.069(0.433 ; 0.176,1.066)$ & $\mathrm{NP}$ \\
T stage & $0.312(0.892 ; 0.716,1.113)$ & $0.274(1.140 ; 0.901,1.442)$ \\
$D_{\max }$ & $0.859(0.991 ; 0.899,1.093)$ & $0.549(1.028 ; 0.938,1.127)$ \\
\hline
\end{tabular}

Dmax maximum diameter of the lesion, $N P$ not performed 
Although colonoscopy is considered the gold standard in the detection of colorectal lesions, little is known about its accuracy in lesion localization [1].

Additionally, it has not been extensively clarified whether CT scans could be considered an aid to correctly localize colonic lesions $[6,9]$.

Several publications have demonstrated variability in the accuracy of colonoscopy with a range from 79 to $88 \%$ [3-5, 13-15] that decreases to $63.5 \%$ in the transverse colon [16].

In contrast, few studies have investigated the accuracy of $\mathrm{CT}$ in determining lesion location, reporting an overall accuracy ranging from 42.3 to $90.5 \%[6,10,16,17]$.

However, in recent years, the accuracy of CT and colonoscopy in localizing lesions has been questioned $[6,10$, 16-19].

Lee et al. [6], in a retrospective analysis of 104 patients affected by colon cancer, reported an accuracy of $79.8 \%$ for colonoscopy and 50\% for CT, with missed lesions in $32.7 \%$ of cases.

Similarly, Feuerlein et al. [10] analysed data from 46 patients and demonstrated that conventional colonoscopy and CT imaging had an accuracy of $78.7 \%$ and $67.4 \%$ in localizing colonic lesions, respectively.

A lower accuracy rate was observed by Solon et al. [16] in their analysis of 101 patients with right colon cancer. In fact, the author reported an overall accuracy of $43 \%$ for CT and $59.5 \%$ for endoscopy.

In contrast, higher accuracy rates for colonoscopy and CT scans in localizing sigmoidal and rectal lesions was demonstrated by Loffeld et al. [17]. The author reported an overall accuracy of $87.5 \%$ for colonoscopy and $90.5 \%$ for imaging.

More recently, Johnstone et al. [18] demonstrated in a prospective multicentric analysis of 79 patients with colorectal cancer that colonoscopy accurately located $81 \%$ of tumours, while CT was unable to identify the primary tumour in $23.1 \%$ of cases, with an overall accuracy of $88.3 \%$ among cases in which the lesion was detected.

Finally, Moug et al. [19] analysed 364 patients with colorectal cancer in a large prospective study and demonstrated an overall accuracy of $82 \%$ for colonoscopy and 59\% for CT. However, when considering only the lesions that could be seen on CT scans, the accuracy increased to $80 \%$.

Considering our results, the accuracy of tumour localization is in the range reported in the current literature.

In fact, based on the data of 729 patients, colonoscopy and CT scans were accurate in $74.6 \%$ and $70.1 \%$ of cases, respectively. However, when considering only the lesions that were detected by CT, the accuracy of this imaging tool in localizing colonic lesions increased to $77.2 \%$.

The comparison between CT and colonoscopy did not show a significant difference in terms of accuracy in localizing visible lesions, even when considering only the visible lesions and the patients who underwent complete colonoscopy. However, when considering the intraoperative localization results as the reference standard, the comparison between the two preoperative tools showed that colonoscopy significantly failed to correctly locate the lesions in the descending colon.

This result is in accordance with the current literature, which has demonstrated that colonoscopy has a lower accuracy in localizing lesions in the colonic segments far from anatomic landmarks [16].

By analysing the potential impact of risk factors on incorrect localization, we showed that none of the patients' characteristics or pathologic data significantly influenced lesion localization with either preoperative tool. Our results are in contrast with the current literature, which has proposed several risk factors $[3,4,8,15]$.

Vaziri et al. [3], in their analysis of 374 patients, proposed increased age as a potential risk factor for incorrect lesion localization but found that patient sex did not significantly impact lesion localization.

In contrast, Piscatelli et al. [15] and Borda et al. [4] showed that age did not impact lesion localization, but they demonstrated that the significant influencing factors were previous abdominal surgery and incomplete colonoscopy, respectively.

Finally, Bryce et al. [8] noted incomplete colonoscopy as a unique risk factor for incorrect lesion localization.

To the best of our knowledge, this is the largest series to compare CT and conventional colonoscopy in lesion localization.

However, a major limitation of the study has to be addressed. Indeed, as a multicentric study, the different experiences of the involved specialists among the centres could represent an important concern.

In conclusion, we did not identify that $\mathrm{CT}$ has an advantage in localizing colonic tumours. In this setting, colonoscopy should be considered the standard reference to properly localize the lesions; however, to better localize lesions in the descending colon, CT could be considered a valuable tool to improve the accuracy of lesion localization.

\section{Acknowledgements PLoCoS Study group}

Giovanni Sarnelli, Nicola Gennarelli and Sara Vertaldi (Department of Clinical Medicine and Surgery, "Federico II" University of Naples, Via Pansini 5, 80131 Naples, Italy); Giuseppe Sammarco (Department of Health Sciences, O.U. of Digestive Surgery, University of Catanzaro, Catanzaro, Italy), Giuseppina Vescio and Vincenzo Tiesi (Operative Unit of General Surgery, Department of Medical and Surgical Sciences, University of Catanzaro), Francesco Pata (Operative Unit of General Surgery, Department of Medical and Surgical Sciences, University of Catanzaro; General Surgery Unit, Nicola Giannettasio Hospital, Corigliano-Rossano, Italy); Donato Francesco Altomare, Arcangelo Picciariello and Vincenzo Papagni (Department of Emergency and Organ Transplantation, University "Aldo Moro", Bari, Italy), Leonardo Vincenti (Surgical Unit, Azienda Ospedaliero-Universitaria Policlinico Bari, Bari, Italy); Massimiliano Mistrangelo, Edoardo Forcignanò and Antonio Salzano (Department of Surgical Sciences, University 
of Turin, 10126 Turin, Italy); Andrea Bondurri, Anna Maffioli and Francesco Colombo (Department of General Surgery, Luigi Sacco University Hospital, ASST FBF-Sacco, Milan, Italy); Andrea Lauretta (Unit of General Oncologic Surgery, Centro di Riferimento Oncologico di Aviano (CRO), IRCCS Aviano, Aviano, Italy); Giuseppe Sica and Michela Campanelli (Department of Surgical Science, University Hospital Tor Vergata, Rome, Italy); Marco Stella, Paolo Boati and Francesco Ferrara (Department of Surgery, San Carlo Borromeo Hospital, ASST Santi Paolo e Carlo, Milan, Italy); Francesco Selvaggi, Gianluca Pellino, Francesco Maria Romano and Lucio Selvaggi (Colorectal Surgery, Department of Advanced Medical and Surgical Sciences, Universita' degli Studi della Campania Luigi Vanvitelli, Naples, Italy); Yves Panis, Alice Frontali and Giovanni Spiezio (Department of Colorectal Surgery, Pôle des Maladies de l'Appareil Digestif (PMAD), Beaujon Hospital, Assistance publique-Hôpitaux de Paris (AP-HP), University Denis Diderot (Paris VII), 100 boulevard du Général Leclerc, 92118, Clichy cedex, France.); Antonino Spinelli (Department of Biomedical Sciences, Humanitas University, Via Rita Levi Montalcini 4, 20090 Pieve Emanuele-Milan, Italy; IRCCS Humanitas Research Hospital, Via Manzoni 56, 20089 Rozzano-Milan, Italy), Francesca Di Candido and Annalisa Maroli (IRCCS Humanitas Research Hospital, Via Manzoni 56, 20089 Rozzano-Milan, Italy); Claudio Coco and Gianluca Rizzo (U.O.C. Chirurgia Generale 2, Fondazione Policlinico Universitario "Agostino Gemelli"-IRCCS, Università Cattolica del Sacro Cuore, Rome, Italy); Elisabetta Moggia (Department of General Surgery, Infermi Hospital, Rivoli, Torino, Italy); Gaetano Luglio, Gianluca Pagano and Francesca Paola Tropeano (Department of Public Health, Endoscopic Surgery Unit, "Federico II" University, Via Sergio Pansini 5, 80131 Naples, Italy) and Roberto Peltrini (Department of Clinical Medicine and Surgery, "Federico II" University of Naples, Via Pansini 5, 80131 Naples, Italy); Federico Marchesi, Gabriele Luciano Petracca, Giorgio Dalmonte and Marina Valente (Unit of General Surgery, Parma University Hospital, Parma, Italy), Antonio Giuliani (Department of Biotechnological and Applied Clinical Sciences, San Salvatore Hospital, L'Aquila, Italy); Harmony Impellizzeri, Enrico Marrano and Gianluigi Moretto (Operative Unit of General Surgery-Pederzoli Hospital, Peschiera del Garda (VR), Italy); Cristina Folliero (Operative Unit of General Surgery AOU Santa Maria della Misericordia, Udine, Italy); Antonio Langone and Giuseppe Caristo (Operative Unit of General Surgery San Paolo Hospital, Savona, Italy); Giorgio Maria Paolo Graziano, Angelo Amico and Antonio Di Cataldo (General Surgery, Department of Oncological Surgery, University Hospital "PoliclinicoVittorio Emanuele", 95123 Catania, Italy); Pietro Maida, Ester Marra and Roberta Abete (General Surgery Unit, Del Mare Hospital, Naples, Italy); Antonio Castaldi, Alessio Palumbo, Fabrizio Foroni and Carmine Antropoli (Department of Gastroenterological Surgery, III Surgery, A. Cardarelli Hospital, Naples, Italy); Paola De Nardi, Roberto Quattromani and Riccardo Rosati (Division of Gastrointestinal Surgery, San Raffaele Scientific Institute, Milan, Italy).

Author contribution Conceptualization: MMa; Methodology: MMa, MMi, MMu, PV, FM, UE, GG; Formal analysis and investigation: all Authors; Writing — original draft preparation: MMa, MMi, GG; Writing - review and editing: MMa, MMi, MMu, PV, FM, UE, GG, RP, GDP; Supervision: RP, GDP; Final Approval: All Authors.

Funding Open access funding provided by Università degli Studi di Napoli Federico II within the CRUI-CARE Agreement. None.

Availability of data and materials Data are available to the corresponding Author.

\section{Declarations}

Conflict of interest The authors declare that they have no conflicts of interest.

Ethical approval All procedures performed in studies involving human participants were in accordance with the ethical standards of the institutional and/or national research committee and with the 1964 Helsinki Declaration and its later amendments or comparable ethical standards.

Consent to participate Informed consent was obtained from all participants included in the study.

Consent for publication Informed consent for publication was obtained from all participants included in the study.

Open Access This article is licensed under a Creative Commons Attribution 4.0 International License, which permits use, sharing, adaptation, distribution and reproduction in any medium or format, as long as you give appropriate credit to the original author(s) and the source, provide a link to the Creative Commons licence, and indicate if changes were made. The images or other third party material in this article are included in the article's Creative Commons licence, unless indicated otherwise in a credit line to the material. If material is not included in the article's Creative Commons licence and your intended use is not permitted by statutory regulation or exceeds the permitted use, you will need to obtain permission directly from the copyright holder. To view a copy of this licence, visit http://creativecommons.org/licenses/by/4.0/.

\section{References}

1. Gamaleldin M, Benlice C, Delaney CP et al (2018) Management of the colorectal polyp referred for resection: a case-matched comparison of advanced endoscopic surgery and laparoscopic colectomy. Surg (United States). https://doi.org/10.1016/j.surg. 2017.10.057

2. Gallo G (2020) Preoperative Colorectal-Cancer Detection: Do We Need Anything Else? An Invited Brief Commentary on Is CT Scan More Accurate than Endoscopy in Identifying Distance from the Anal Verge for Left-sided Colon Cancer? A Comparative Cohort Analysis. J Investig Surg

3. Vaziri K, Choxi SC, Orkin BA (2010) Accuracy of colonoscopic localization. Surg Endosc. https://doi.org/10.1007/ s00464-010-0993-2

4. Borda F, Jiménez FJ, Borda A et al (2012) Endoscopic localization of colorectal cancer: study of its accuracy and possible error factors. Rev Española Enfermedades Dig. https://doi.org/10.4321/ s1130-01082012001000002

5. Stanciu C, Trifan A, Khder SA (2007) Accuracy of colonoscopy in localizing colonic cancer. Rev medico-chirurgicală a Soc Medici şi Nat din Iaşi

6. Lee J (2010) Accuracy of colon tumor localization: computed tomography scanning as a complement to colonoscopy. World J Gastrointest Surg. https://doi.org/10.4240/wjgs.v2.i1.22

7. Elarini T, Wexner SD, Isenberg GA (2015) The need for standardization of colonoscopic tattooing of colonic lesions. Dis Colon Rectum

8. Bryce AS, Johnstone MS, Moug SJ (2015) Improving lesion localisation at colonoscopy: an analysis of influencing factors. Int J Colorectal Dis. https://doi.org/10.1007/s00384-014-2052-2 
9. Azin A, Saleh F, Cleghorn M et al (2017) A comparison of endoscopic localization error rate between operating surgeons and referring endoscopists in colorectal cancer. Surg Endosc. https:// doi.org/10.1007/s00464-016-5114-4

10. Feuerlein S, Grimm LJ, Davenport MS et al (2012) Can the localization of primary colonic tumors be improved by staging CT without specific bowel preparation compared to optical colonoscopy? Eur J Radiol. https://doi.org/10.1016/j.ejrad.2011.12.004

11. von Elm E, Altman DG, Egger M et al (2008) The strengthening the reporting of observational studies in epidemiology (STROBE) statement: guidelines for reporting observational studies. J Clin Epidemiol. https://doi.org/10.1016/j.jclinepi.2007.11.008

12. Acuna SA, Elmi M, Shah PS et al (2017) Preoperative localization of colorectal cancer: a systematic review and meta-analysis. Surg Endosc. https://doi.org/10.1007/s00464-016-5236-8

13. Cho YB, Lee WY, Yun HR et al (2007) Tumor localization for laparoscopic colorectal surgery. World J Surg. https://doi.org/10. 1007/s00268-007-9082-7

14. Lam DTY, Kwong KH, Lam CW et al (1998) How useful is colonoscopy in locating colorectal lesions? Surg Endosc. https://doi. org/10.1007/s004649900725

15. Piscatelli N, Hyman N, Osler T (2005) Localizing colorectal cancer by colonoscopy. Arch Surg. https://doi.org/10.1001/archsurg. 140.10 .932
16. Solon JG, Al-Azawi D, Hill A et al (2010) Colonoscopy and computerized tomography scan are not sufficient to localize rightsided colonic lesions accurately. Color Dis. https://doi.org/10. 1111/j.1463-1318.2009.02144.x

17. Loffeld RJLF, Flens M, Fransen G et al (2014) The localisation of cancer in the sigmoid, rectum or rectosigmoid junction using endoscopy or radiology — what is the most accurate method? J Gastrointest Oncol. https://doi.org/10.3978/j.issn.2078-6891. 2014.087

18. Renwick A, Vella M, Porteous C et al (2014) The accuracy of colonoscopic localization of colorectal tumours: a prospective, multi-centred observational study. Scott Med J. https://doi.org/ 10.1177/0036933014529051

19. Moug SJ, Fountas S, Johnstone MS et al (2017) Analysis of lesion localisation at colonoscopy: outcomes from a multi-centre U.K. study. Surg Endosc. https://doi.org/10.1007/s00464-016-5313-z

Publisher's Note Springer Nature remains neutral with regard to jurisdictional claims in published maps and institutional affiliations. 